\title{
Érythème au site d'une cicatrice du vaccin bacille de Calmette-Guérin après l'obtention d'une dose du vaccin à ARN messager contre le SRAS-CoV-2
}

\author{
Darren Wan-Teck Lim MRCP, Dorothy Hui Lin Ng MBBS-PhD MRCP, Jenny Guek-Hong Low MRCP MSP
}

- Citation : CMAJ 2021 August 3;193:E1178. doi : 10.1503/cmaj.210696-f

Voir la version anglaise de l'article ici : www.cmaj.ca/lookup/doi/10.1503/cmaj.210696

$\mathbf{U}$ ne femme de 34 ans s'est présentée pour une consultation externe avec un œdème et un érythème au site d'une cicatrice du vaccin bacille de Calmette-Guérin (BCG) obtenu dans son enfance, 7 jours après avoir reçu sur le bras ipsilatéral sa première dose du vaccin à acide ribonucléique messager (ARN messager) BNT162b2 (PfizerBioNTech) contre le SRAS-CoV-2 (figure 1A). Elle a signalé une myalgie et de la fatigue associées au vaccin. Le site d'injection du vaccin BNT162b2 n'était pas enflammé. On a administré la deuxième dose du vaccin sans incident.

Une femme de 45 ans s'est présentée pour une consultation externe avec un œdème et un érythème au site de la cicatrice de son vaccin BCG sur le bras controlatéral, 4 jours après avoir obtenu sa deuxième dose du vaccin à ARN messager BNT162b2 (figure 1B). Le site d'injection du vaccin BNT162b2 n'était pas enflammé et elle a obtenu sa seconde dose sans incident.

Dans les 2 cas, l'œdème et l'érythème présents au site de la cicatrice du vaccin BCG se sont résorbés de façon spontanée dans les 7 jours suivants leur apparition, sans autre traitement.

On a récemment signalé des cas sporadiques d'inflammation du site de la cicatrice du vaccin BCG après l'administration d'une deuxième dose des vaccins à ARN messager mRNA-1273 (Moderna) et BNT162b2. Ces cas ont suivi une évolution clinique comparable à celle de nos patientes ${ }^{1}$. Le mécanisme de l'inflammation demeure inconnu. Nous supposons qu'elle pourrait être le résultat de la stimulation passive des cellules $T$ induite par l'activation immunitaire associée au vaccin. On a déjà décrit la réactivation des cicatrices du vaccin BCG dans le cadre de la vaccination contre la grippe, de la maladie infantile de Kawasaki et d'infections virales ${ }^{2,3}$; il est ainsi possible que des vaccins contre le SRAS-CoV-2 qui ne font pas appel à des ARN messagers puissent engendrer des réactions similaires. La réactivité immunitaire croisée des nanoparticules lipidiques présentes dans les vaccins à base d'ARN messager contre les parois cellulaires mycobactériennes riches en lipides est plausible, bien que nous n'ayons pas pris connaissance de données qui suggèrent qu'un tel mimétisme moléculaire puisse se produire.

Ce phénomène fait probablement l'objet d'une sous-déclaration. Cependant, les érythèmes au site des cicatrices du vaccin BCG à la suite de l'administration des vaccins à ARN messager contre le SRAS-CoV-2 semblent être bénins, comme dans les cas présentés ici, et elles ne devraient pas inquiéter les médecins, limiter la vaccination, ni être un argument justifiant la réticence à l'égard de la vaccination. 


\section{Références}

1. Lopatynsky-Reyes EZ, Acosta-Lazo H, Ulloa-Gutierrez R, et al. BCG scar local skin inflammation as a novel reaction following mRNA COVID-19 vaccines in two international healthcare workers. Cureus 2021;1313:e14453.

2. Chavarri-Guerra Y, Soto-Perez-de-Celis E. Erythema at the bacillus Calmette-Guerin scar after influenza vaccination. Rev Soc Bras Med Trop 2019;53:e20190390. doi: 10.1590/0037-8682-0390-2019.

3. Kakisaka Y, Ohara T, Katayama S, et al. Human herpes virus type 6 can cause skin lesions at the BCG inoculation site similar to Kawasaki disease. Tohoku J Exp Med 2012;228:351-3.

\section{Intérêts concurrents : Aucun déclaré.}

Cet article a été révisé par des pairs.

Les auteurs ont obtenu le consentement des patientes.

Affiliations : École des sciences infirmières de l'Université Duke (Lim); Hôpital général de Singapour (Ng); Programme des maladies infectieuses émergentes (Low); École des sciences infirmières de l'Université Duke, Maladies infectieuses (Low), Hôpital général de Singapour, Singapour.

Propriété intellectuelle du contenu : Il s'agit d'un article en libre accès distribué conformément aux modalités de la licence Creative Commons Attribution (CC BY-NC-ND 4.0), qui permet l'utilisation, la diffusion et la reproduction de tout médium à la condition que la publication originale soit adéquatement citée, que l'utilisation se fasse à des fins non commerciales (c.-à-d., recherche ou éducation) et qu'aucune modification ni adaptation n'y soit apportée. Voir : https://creativecommons.org/licenses/by-nc-nd/4.0/deed.fr.

Correspondance : Darin Wan-Teck Lim, dmolwt@nccs.com.sg

Les images cliniques sont choisies pour leur caractère particulièrement intéressant, classique ou impressionnant. Toute soumission d'image de haute résolution claire et bien identifiée doit être accompagnée d'une légende aux fins de publication. On demande aussi une brève explication (300 mots maximum) de la portée éducative des images, et des références minimales. Le consentement écrit du patient au regard de la publication doit être obtenu avant la soumission. 